\title{
Disturbance Rejection Control of an Electromagnetic Bearing Spindle
}

\author{
Rebecca Petteys \\ Gordon Parker \\ Michigan Technological University \\ Department of Mechanical Engineering \\ 1400 Townsend Dr. \\ Houghton, MI 49931 \\ James Redmond \\ Sandia National Laboratories \\ Albuquerque, NM 87111
}

\section{ASTRACT}

The force exerted on the rotor by an active magnetic bearing (AMB) is determined by the current flow in the magnet coils. This force can be controlled very precisely, making magnetic bearings a potential benefit for grinding, where cutting forces act as external disturbances on the shaft, resulting in degraded part finish. It is possible to achieve precise shaft positioning, reduce vibration of the shaft caused by external disturbances, and even damp out resonant modes. Adaptive control is an appealing approach for these systems because the controller can tune itself to account for an unknown periodic disturbance, such as cutting or grinding forces, injected into the system. In this paper we show how one adaptive control algorithm can be applied to an AMB system with a periodic disturbance applied to the rotor. An adaptive algorithm was developed and implemented in both simulation and hardware, yielding significant reductions in rotor displacement in the presence of an external excitation. Ultimately, this type of algorithm could be applied to a magnetic bearing grinder to reduce unwanted motion of the spindle which leads to poor part finish and chatter.

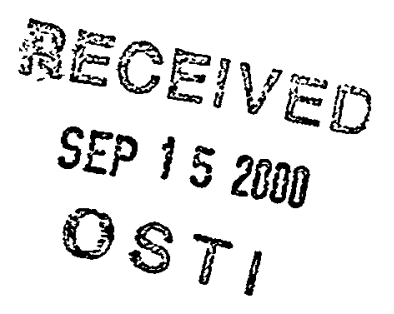

\author{
Spindle
}

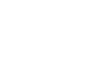




\section{DISCLAIMER}

This report was prepared as an account of work sponsored by an agency of the United States Government. Neither the United States Government nor any agency thereof, nor any of their employees, make any warranty, express or implied, or assumes any legal liability or responsibility for the accuracy, completeness, or usefulness of any information, apparatus, product, or process disclosed, or represents that its use would not infringe privately owned rights. Reference herein to any specific commercial product, process, or service by trade name, trademark, manufacturer, or otherwise does not necessarily constitute or imply its endorsement, recommendation, or favoring by the United States Government or any agency thereof. The views and opinions of authors expressed herein do not necessarily state or reflect those of the United States Government or any agency thereof. 


\section{DISCLAIMER}

Portions of this document may be illegible in electronic image products. Images are produced from the best available original document. 
metal cutting processes, where cutting forces act as external disturbances on the shaft, resulting in degraded part finish. AMB systems also do not have the wear and lubrication problems seen by conventional bearings, but require additional electronic and cooling systems. Furthermore, they are limited in load capability

Adaptive control is an appealing approach for these systems because the controller can tune itself to account for an unknown periodic disturbance, such as cutting forces injected into the system. In this paper we show how one adaptive control algorithm can be applied to an AMB system with a periodic disturbance applied to the rotor. The purpose is to create an input signal that would counteract the disturbance and result in minimal motion of the spindle. In an application such as grinding, this would result in improved part finish, reduction of chatter, etc. First a model of the experimental system must be developed to test the proposed control strategy. Then the adaptive control algorithm will be described and results will be shown.

\section{EXPERIMENTAL SETUP}

The bearing system modelled and used for this paper is a modified MBC500 from Magnetic Moments, Inc. The MBC500 has two active magnetic bearings, each consisting of four electromagnets, supporting the spindle. The bearings are mounted on top of an anodized aluminum case which houses the electronics and also acts as a heat sink for the magnets. The spindle is actively positioned in the radial direction at the bearings and freely rotates about its long axis. The front panel shows a block diagram of the system with BNC connections for easy access to system inputs and outputs. An air turbine drives the shaft to speeds up to 10,000 RPM.

The system has four on-board analog lead-filter controllers that levitate the spindle in its default mode. These controllers can be disabled with switches on the front panel, allowing an external controller to be implemented. The BNC connections also allow for an external controller to be wrapped around the on-board controllers while they are still engaged. The modified version of the $\mathrm{MBC} 500$ include an external electromagnet mounted on the case near the center of the spindle to be used as a disturbance source. The magnet can be moved to vary the gap between the magnet and the spindle, thereby allowing for a large range of applied forces. The current flow to this magnet is controlled by an external amplifier.

\section{SYSTEM MODEL}

An accurate model of the system is necessary for designing and testing prospective control strategies. Magnetic bearings are highly nonlinear by nature and. in order to best capture the effects of those nonlinearities. a Simulink simulation was designed. A block diagram representation of the system is shown in Figure 1. This diagram represents the MBC500 with- out any external controllers, but with the external magnet applying a disturbance force to the spindle. It is assumed that the spindle is not spinning, so there are no gyroscopic effects and the axes are completely uncoupled. The external force is assumed to be applied exactly at the center of the shaft. The system inputs and outputs are also shown.

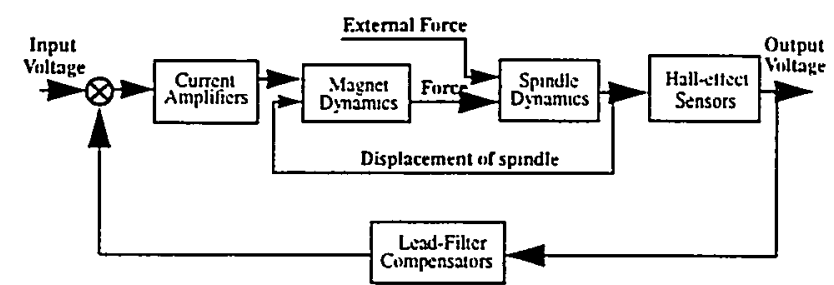

Figure 1. Simulink model used to simulate the magnetic bearing system

When external controllers are implemented, either the feedback loop containing the lead-filter compensators is broken and replaced with a computer system which implements the digital controllers, or an external controller is wrapped around the onboard compensators, leaving the feedback loop intact. For the implementation of the adaptive control algorithm presented in this paper, the on-board controllers are left intact and the external adaptive controller is wrapped around the feedback loop.

There are five main components to the system that must be included in the model: the spindle dynamics, the magnet dynamics, the on-board controllers, the current amplifiers, and the position sensors. Each is dealt with in the following sections. Definitions of the variables and parameters used in the description of the model and system are given in Table 1 and Table 2

\section{Spindle Dynamics}

Both the rigid and the first two flexible body modes were incorporated into the simulation. Because the axes are uncoupled, we

Table 1: Definitions of variables

\begin{tabular}{l|l}
\hline$x_{0}$ & displacement of center of mass of rotor \\
\hline$x_{1}$ and $x_{2}$ & displacement of rotor at left and right bearings \\
\hline$X_{1}$ and $X_{2}$ & displacement of rotor at Hall Effect sensors \\
\hline$\theta$ & tilt of rotor about $y$-axis \\
\hline$F_{1}$ and $F_{2}$ & forces exened on rotor at left and right bearings \\
\hline$F_{e}$ & external applied force \\
\hline
\end{tabular}


Table 2: Definitions of parameters

\begin{tabular}{|l|l|}
\hline$L=0.269 \mathrm{~m}$ & total length of rotor \\
\hline$l_{1}=0.024 \mathrm{~m}$ & $\begin{array}{l}\text { distance from each bearing to end of } \\
\text { rotor }\end{array}$ \\
\hline$l_{2}=0.0028 \mathrm{~m}$ & $\begin{array}{l}\text { distance from each sensor to end of } \\
\text { rotor }\end{array}$ \\
\hline$l_{0}=1.5884 \times 10^{-3} \mathrm{~kg} \mathrm{~m}^{2}$ & $\begin{array}{l}\text { moment of inertia of rotor around } \mathrm{y}- \\
\text { axis }\end{array}$ \\
\hline$m=0.2629 \mathrm{~kg}$ & mass of rotor \\
\hline$a=0.0107 \mathrm{~m}$ & $\begin{array}{l}\text { distance from bearing } 1 \text { to external } \\
\text { magnet }\end{array}$ \\
\hline$b=0092 \mathrm{~m}$ & $\begin{array}{l}\text { distance from bearing } 2 \text { to external } \\
\text { magnet }\end{array}$ \\
\hline
\end{tabular}

may look at each independently and can assume that the dynamics in each direction are the same (with the exception of the constant force of gravity in the y-direction). On the experimental set-up, the external force is applied in only the $x$-direction, so the equations of motion will be derived only for the $x$-direction.

Rigid Body Dynamics. For rigid body motion, the spindle is assumed to move without bending. (Flexible body motion will be considered in the next section.) Figure 2 shows the spindle from above. The coordinate system and variables are defined as shown.

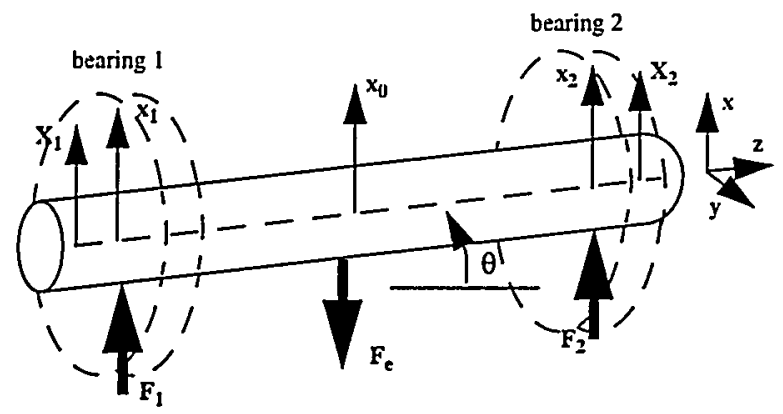

Figure 2. The spindle shown with variables defined.

The axis are uncoupled, so, assuming the spindle is radially symmetric, the $x$ - and $y$-axes will have the same equations of motion (again, with the exception of the constant force of gravity in the $y$-direction). As shown in the figure, the sensors are located just outside the bearings along the spindle and the external force, $F_{e}$, is applied near the center of mass of the spindle in the negative $\mathrm{x}$-direction.
There are two rigid body degrees of freedom for each axis. This means that there must be two independent coordinates chosen to describe the system. In this case, it is easiest to choose the position of the center of mass of the spindle, $x_{0}$, and the angle of rotation of the spindle from the equilibrium position, $\theta$. The equations of motion for the rigid body motion in the $x$-direction are found by balancing the forces and moments about the center of the shaft.

$$
\begin{gathered}
\sum F=m \ddot{x}_{0}=F_{1}+F_{2}-F_{e} \\
\sum M_{0}=I_{0} \ddot{\theta}=\left(F_{2}-F_{1}\right)\left(\frac{L}{2}-l_{1}\right)+\frac{1}{2} F_{e}(b-a)
\end{gathered}
$$

The clearance at the bearings is $\pm 0.4 \mathrm{~mm}$, so small-angle approximations are appropriate. For rigid body motion, the only restoring forces are those exerted by the magnets, which are nonlinear and cannot be included in the linear rigid body statespace model. Therefore, the state-space system is constructed such that the forces -- the external force and the bearing forces on either end of the spindle -- are the inputs and the displacements of the spindle at the bearings and sensors are the outputs. The state vector is chosen to consist of the displacement of the center of mass of the spindle, the angle of rotation and their respective time derivatives. The resulting system is shown below.

$$
\begin{gathered}
\dot{X_{R B}}=A_{R B} X_{R B}+B_{R B} U_{R B} \\
Y_{R B}=C_{R B} X_{R B}
\end{gathered}
$$

where

$$
X_{R B}=\left[\begin{array}{c}
x_{0} \\
\dot{x}_{0} \\
\theta \\
\dot{\theta}
\end{array}\right], U_{R B}=\left[\begin{array}{l}
F_{1} \\
F_{2} \\
F_{e}
\end{array}\right], Y_{R B}=\left[\begin{array}{c}
X_{1} \\
x_{1} \\
X_{2} \\
x_{2}
\end{array}\right]
$$

$$
\begin{aligned}
& A_{R B}=\left[\begin{array}{llll}
0 & 1 & 0 & 0 \\
0 & 0 & 0 & 0 \\
0 & 0 & 0 & 1 \\
0 & 0 & 0 & 0
\end{array}\right], B_{R B}=\left[\begin{array}{ccc}
0 & 0 & 0 \\
\frac{1}{m} & \frac{1}{m} & \frac{1}{m} \\
0 & 0 & 0 \\
-\frac{1}{I_{0}}\left(\frac{L}{2}-l_{1}\right) \frac{1}{I_{0}}\left(\frac{L}{2}-l_{1}\right) & 0
\end{array}\right], \\
& C_{R B}=\left[\begin{array}{cccc}
1 & 0 & -\left(L / 2-l_{2}\right) & 0 \\
1 & 0 & -\left(L / 2-l_{1}\right) & 0 \\
1 & 0 & \left(L / 2-l_{2}\right) & 0 \\
1 & 0 & \left(L / 2-l_{1}\right) & 0
\end{array}\right]
\end{aligned}
$$


where 'RB' signifies the rigid body matrices and states. The output of the system depends on the sine of the tilt angle, which has been approximated as unity.

Flexible Body Dynamics. Only the first two bending modes are considered for this simulation. The mass and stiffness matrices for the shaft are taken from the MBC500 manual and are essentially the free-free modes of the shaft and damping was found experimentally to be approximately $1.3 \%$ for the first bending mode and $0.15 \%$ for the second bending mode. The modal equation for the $\mathrm{x}$-direction in matrix form is then

$$
M \ddot{a}+C \dot{a}+K a=P
$$

The state-space system for the flexible body dynamics can be developed in the same manner as the rigid body equations.

They are expressed here as

$$
\begin{gathered}
X_{F B}^{\cdot}=A_{F B} X_{F B}+B_{F B} F_{F B} \\
Y_{F B}=C_{F B} X_{F B}
\end{gathered}
$$

The inputs and outputs for this state-space system must be the same as the rigid body system, but the state vectors will be different.

The total displacement of the shaft is the combined contribution of the rigid and flexible body displacements. In other words,

$$
Y_{t o t}=Y_{R B}+Y_{F B}
$$

This displacement is then the input for the position sensors.

\section{Hall Effect Sensor Dynamics}

The sensors for this system are two orthogonal Hall-effect sensors at each shaft end. The sensors are actually located closer to the ends of the shaft than the bearings (see Table 2 and Figure 2) but we will make the approximation that they are collocated.

The nonlinear sensor output is

$$
V_{\text {sense, }}=5000 X_{i}+24 \times 10^{9} X_{i}^{3}
$$

for $i=1,2$, where $X_{i}$ is measured in meters. Figure 3 shows the relationship between the sensor voltage and the displacement of the shaft. The figure also shows a plot of the above equation linearized about the equilibrium position $\left(X_{i}=0\right)$.

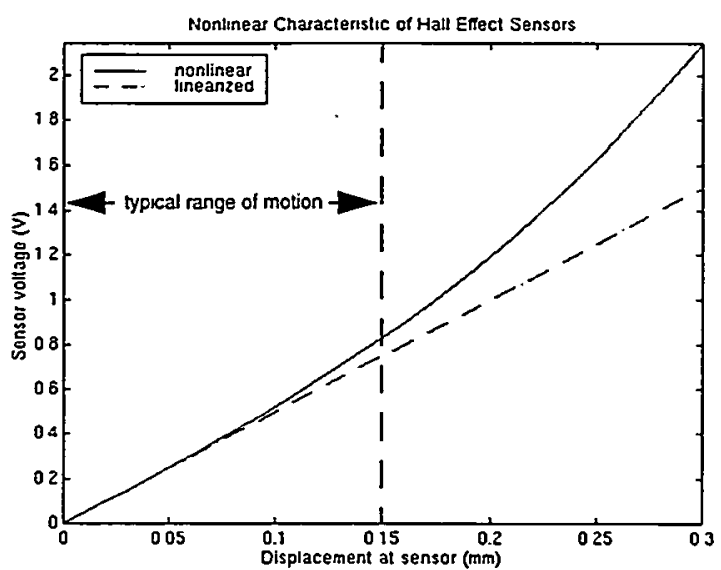

Figure 3. Graph of sensor nonlinearity

As the plot shows, the output is relatively linear for low deflections but the nonlinearity becomes more pronounced with larger displacements $\left(X_{i}>1.5 \times 10^{-1} \mathrm{~m}\right)$. The nonlinear relation is used in the Simulink model, but, as the system usually runs with displacements less than $0.15 \mathrm{~mm}$, the linearized relation could also be validly used.

\section{Lead-Filter Compensator Dynamics}

Since electromagnets can only exert an attractive force, each bearing consists of four magnets, two magnets each in the $x$ and $y$-directions. There is an analog lead-filter compensator for each of the magnet pairs. Each uses the voltage from the corresponding sensor as input and produces an output voltage. The transfer function for the compensators is

$$
V_{\text {control }}=\left(\frac{1.41\left(1+8.9 \times 10^{-4} s\right)}{\left(1+3.3 \times 10^{-4} s\right)\left(1+2.2 \times 10^{-5} s\right)}\right) V_{\text {sense }}
$$

These analog compensators can be removed from the feedback loop so that a digital controller can be implemented. However, for the purposes of this paper, the analog compensators were left in so that the adaptive controller could be implemented without exceeding the limitations of the dSpace components.

\section{Current Amplifier Dynamics}

The actuator current amplifier converts the control voltage to a current for the electromagnet according to

$$
I=\frac{0.25}{1+2.2 \times 10^{-4} s} V_{\text {control }}
$$

The amplifier, in essence. acts as a low-pass filter with a break frequency of, filtering out the very high frequency content of the input signal. 


\section{Electromagnet Dynamics}

The force exerted by one electromagnet on the shaft is

$$
F=\frac{A \mu_{0} N^{2} I^{2}}{4 g^{2}}
$$

where $A$ is the cross-sectional area of the magnet, $\mu_{0}$ is the permittivity of free space, $g$ is the gap between the electromagnet and the rotor, and $N$ is the number of coils in the magnet, each carrying current $l$. For the MBC500 at equilibrium, the gap is $0.0004 \mathrm{~m}$ and there is a bias current of 0.5 amps. If we define $x$ as the displacement from equilibrium and $k$ as $A \mu_{0} N^{2} / 4$ then the total force on the shaft at one bearing due to both magnets (in one direction) is

$$
F_{i}=k \frac{\left(I_{i}-0.5\right)^{2}}{\left(x_{i}+0.0004\right)^{2}}-k \frac{\left(I_{i}+0.5\right)^{2}}{\left(x_{i}-0.0004\right)^{2}}
$$

for $i=1,2$. Note that the force is proportional to the square of the current in the wire and is inversely proportional to the square of the gap width. This represents the strongest nonlinearity in the system. This relationship is plotted in Figure 4 for a range of displacements and currents.

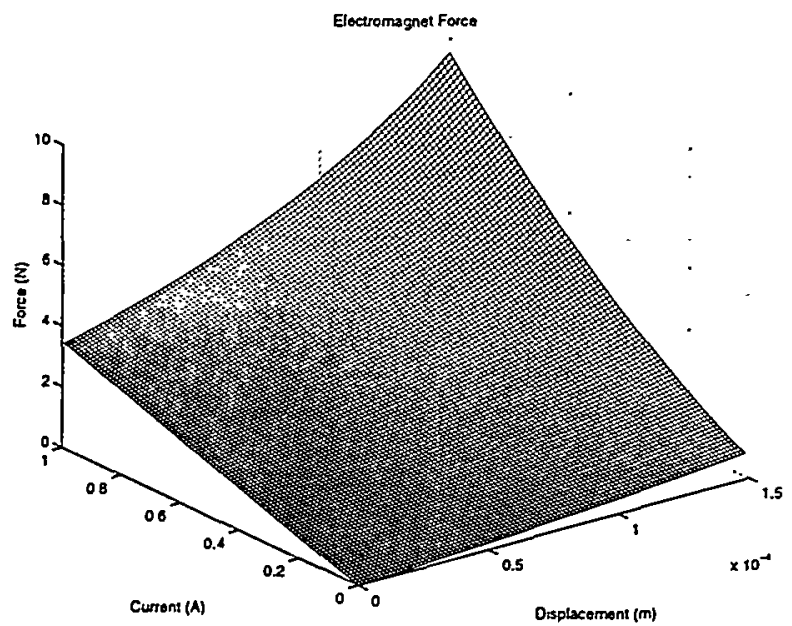

Figure 4. Graph of force exerted by the electromagnets versus current and displacement

As shown in the figure, the output forces become very non-linear and large as the displacement and control current become large. In normal operation, the displacement is rather small $\left(x_{1}<1 \times 10^{-1} \mathrm{~m}\right)$ but the control current may still fluctuate widely because of the dynamic characteristics of the controllers.

\section{Modeling the closed loop system}

Because of the many nonlinearities in the system, a linearized state-space model was not sufficient to model the system accurately. The biggest source of nonlinearity is the magnets, since the force they exert is inversely proportional to the square of the displacement and directly proportional to the square of the control current. Near equilibrium and at steady state, this force can be approximated as linear with a spring constant of $10,500 \mathrm{~N} / \mathrm{m}$, but outside this region $\left(x_{i}>5 \times 10^{-5} \mathrm{~m}\right)$, the approximation is invalid. Another smaller source of nonlinearity is the Hall effect sensors. A linear approximation for these is good to $X_{i}=1.5 \times 10^{-4} \mathrm{~m}$. Because of these limitations, the nonlinear model was used.

For the implementation of the adaptive control algorithm presented below, the on-board analog controllers are left in the feedback loop and the external adaptive controller is wrapped around the feedback loop.

\section{ADAPTIVE CONTROL ALGOTRITHM}

An adaptive controller called the amplitude-phase adaptive control algorithm (APACA) was designed to augment the lead-filter compensator. The purpose of APACA is to predict and compensate for the external disturbance, whereas the purpose of the lead-filter compensator is simply to levitate the spindle.

APACA is based on the MIT Rule. The MIT Rule was one of the first adaptive algorithms and is based on minimizing the cost function

$$
J(\alpha)=\frac{1}{2} e^{2}
$$

where $e$ is the error signal -- in this case, the displacement of the spindle -- and $\alpha$ is the parameter to be varied. This leads to the discrete equation

$$
\alpha(n+1)=\alpha(n)-\gamma e(n) \frac{\partial e}{\partial \alpha}
$$

where $\frac{\partial e}{\partial \alpha}$ is known as the sensitivity derivative.

APACA is designed to be used for external disturbances which are sinusoidal, have a known and fixed frequency, and oscillate between zero and some fixed amplitude. An example of this type of disturbance is grinding. where the force is always in one direction. for example the positive $x$-direction, and varies with the rotational speed of the tool. and so has a constant. known frequency content. Figure 5 shows a block diagram representation of how APACA fits into the total system with the non-adaptive lead-filter compensators. 


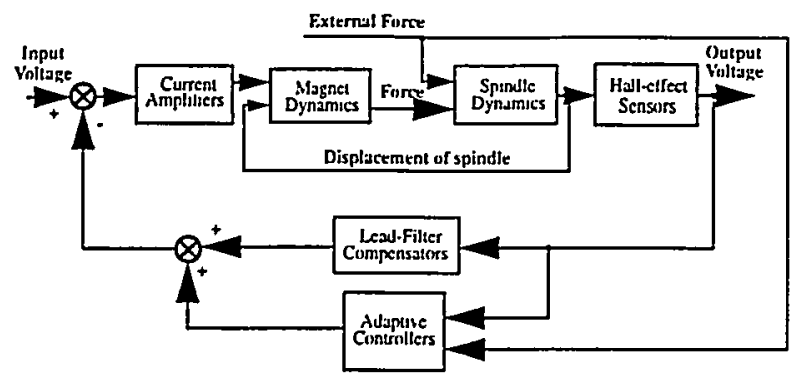

Figure 5. Block diagram including adaptive controller

APACA calculates successive estimates of the amplitude and phase of a complimentary input sine wave that will combine with the disturbance to create zero net motion. The signal from the adaptive controller must go through the amplifier and magnet dynamics before driving the spindle motion through the bearing magnets. Therefore even if the exact disturbance time history is known, it cannot simply be inverted and used directly to cancel itself out. Also, in grinding applications, the amplitude of the disturbance may not be known, but the frequency most likely will be. If the frequency is not known, it can be determined by using an FFT (fast Fourier transform) algorithm on the output signal to determine the dominant frequencies, and then those frequencies can be used in APACA.

Two parameters are varied in determining the output of APACA; the amplitude of the wave $A$ and the phase shift from the disturbance wave (actually computed as a time delay $T$ where $\phi=\omega T$ ). The output then looks like

$$
y_{A}=A(\sin (\omega t+\omega T)+1)
$$

where $\omega$ is the frequency of the disturbance and is assumed to be known.

The two variable parameters are calculated according to a modified MIT Rule. The sensitivity derivative is replaced by the time derivative of the error signal in the time delay equation and by a constant in the amplitude equation (which is absorbed by the constant $\gamma_{d}$ ). The error signal in the time delay equation is replaced by the disturbance signal. These modifications lead to the recursive equations which form the basis of APACA,

$$
\begin{gathered}
A(n+1)=A(n)+\gamma_{A} e(n) \\
T(n+1)=T(n)+\gamma_{T} \frac{d e(n)}{d t} d(n)
\end{gathered}
$$

\section{SIMULATION AND HARWARE}

These equations were implemented in Simulink using the parameters shown in Table 3.

Table 3: Simulation parameters

\begin{tabular}{|l|l|}
\hline \multicolumn{1}{|c|}{ parameter } & \multicolumn{1}{c|}{ value } \\
\hline \hline$\gamma_{A}$ & $1 \times 10^{-5}$ \\
\hline$\gamma_{T}$ & $1 \times 10^{-9}$ \\
\hline sample time & $0.1 \mathrm{~ms}$ \\
\hline
\end{tabular}

These parameters were found through testing the controller in simulation and on the experimental setup and trying to find a balance between short convergence time and stability. Like the MIT Rule, a poor selection for $\gamma_{A}$ and $\gamma_{T}$ (a value that is too large) may result in system instability. However, values that are too small will result in long convergence times and a system that won't be able to adapt to changing disturbances.

\section{RESULTS}

The system was tested in simulation with a disturbance input of $0.5 \mathrm{~N}$ and frequency of $100 \mathrm{~Hz}$. On the hardware, this corresponded to an input current of $2.3 \mathrm{amps}$. The results are shown in the following figures. In order to increase the stability of the system, APACA is not implemented in the simulation until after the transient rigid body motion has been damped out by the onboard analog controllers. The time at which APACA is turned on is marked on the plots. The maximum range of the spindle motion is $\pm 40 \times 10^{-5} \mathrm{~m}$, but normal operating range is $\pm 15 \times 10^{-5} \mathrm{~m}$, so the displacement of the spindle shown in Figure 6 and Figure 7 is near the limit of the normal operating range. There was a small amount of noise injected into the current amplifier signal in the simulation in order to determine its effects on the adaptation algorithm. The power of this noise was determined from steady-state experimental data. 


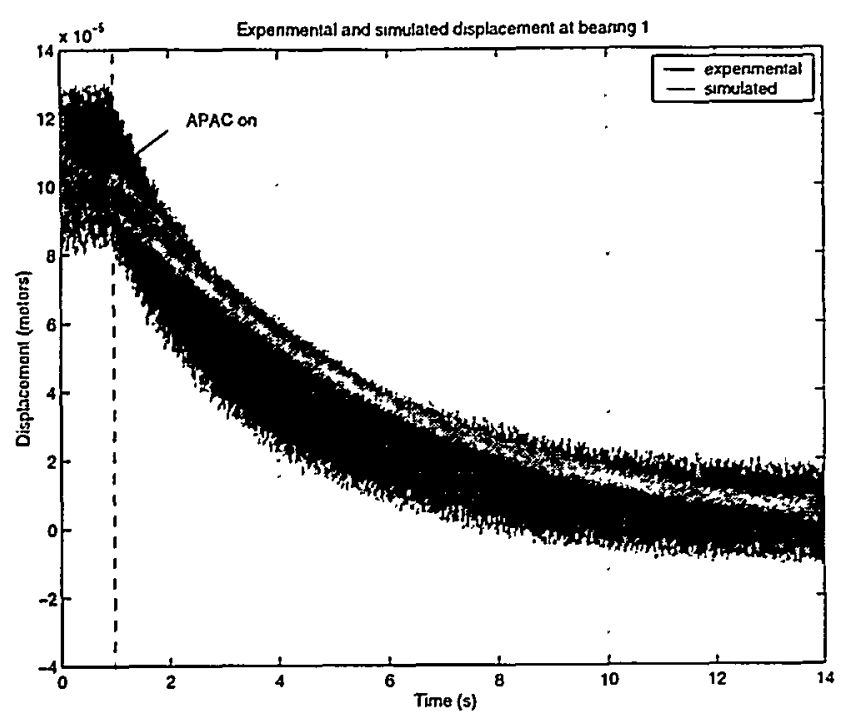

Figure 6. Graph of $\times 1$ displacement with controller on

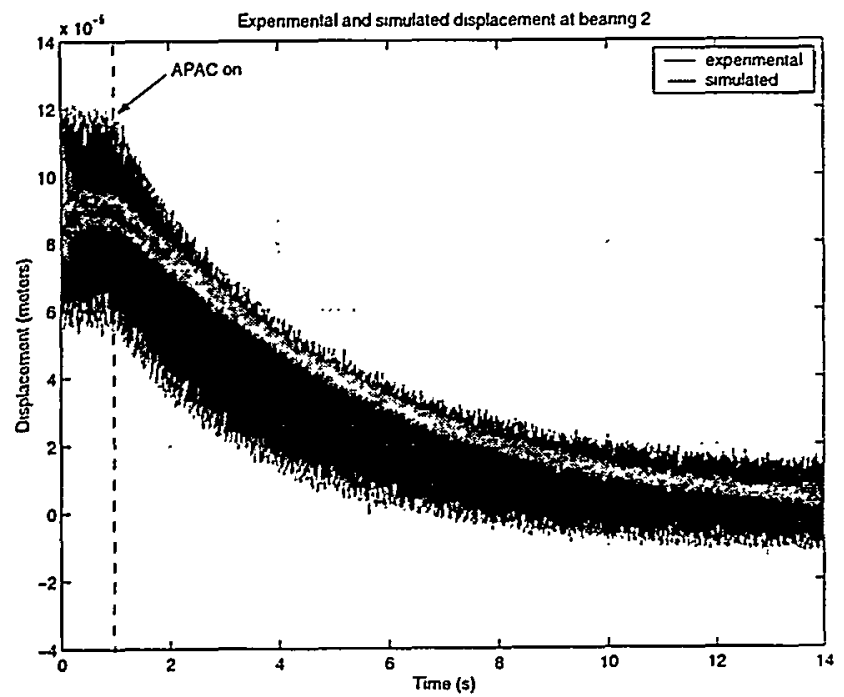

Figure 7. Graph of $x 2$ displacement with controller on

Figure 6 shows the simulated displacement of the spindle at bearing 1 in the $x$-direction and Figure 7 shows the displacement at bearing 2. (Since the external force is not applied at the center of the spindle. the results at bearing 1 and bearing 2 are not identical.) For $0 \leq t<\mid s$, the external force is applied with just the analog lead-filter compensators in place. This results in oscillation about a value offset from zero. At $t=1 \mathrm{~s}$. APACA is turned on and begins to add its signal to the system input. The displacement is quickly reduced and the spindle reaches its equilibrium position approximately 2 seconds after the controller is implemented.

In the simulation, APACA attenuated the amplitude of oscillation at bearing 1 and bearing 2 by $-10.5 \mathrm{~dB}$ and $-8.5 \mathrm{~dB}$ respctively. On the hardware, the oscillation was decreased by -5.3 $\mathrm{dB}$ at bearing 1 and $-7.6 \mathrm{~dB}$ at bearing 2 . These results are summarized in Table 4.

\section{Table 4: Experimental and simulated attenuations of} oscillation amplitude

\begin{tabular}{l|l|l}
\hline & Simulated & Experimental \\
\hline bearing I & $-10.5 \mathrm{~dB}$ & $-5.3 \mathrm{~dB}$ \\
\hline bearing 2 & $-8.5 \mathrm{~dB}$ & $-7.6 \mathrm{~dB}$ \\
\hline
\end{tabular}

The output signal of APACA is shown in Figure 8. In the simulation, the final values to which $A$ and $T$ converge are $0.286 \mathrm{~V}$ and $3.3 \times 10^{-4} \mathrm{~s}$ respectively. This corresponds to an amplitude of $0.25 \mathrm{~N}$ and a phase shift of $0.033 \mathrm{rad}$, or $1.9^{\circ}$. Since there are two bearings acting to counteract the external force, we would expect each to exert a force with an amplitude of half of the external force. The phase shift is due to the dynamics of the magnets and amplifiers.

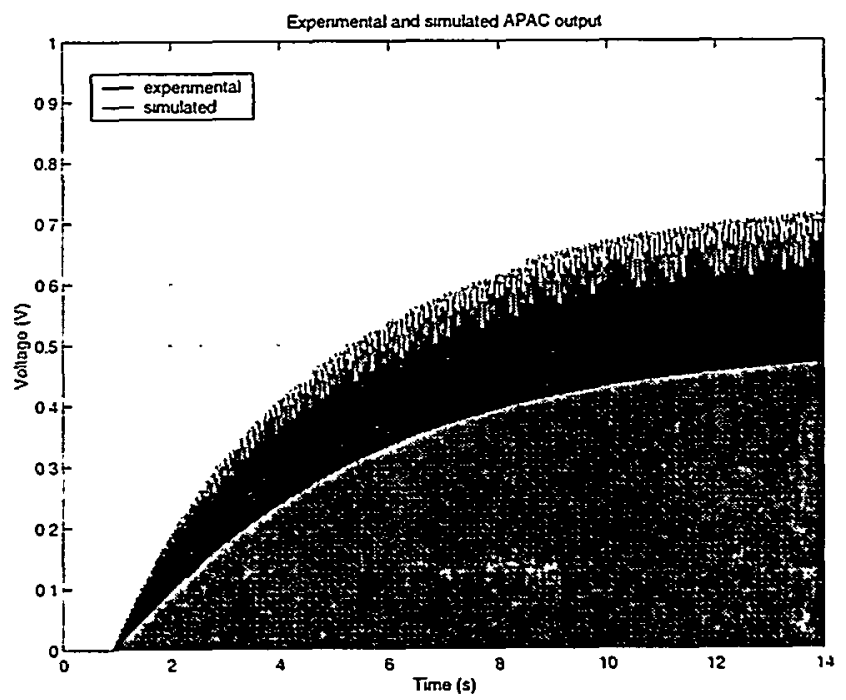

Figure 8. APACA output for experimental and simulation

Due to the complexity and uncertainties of the bearing system. the experimental results did not match with the simulated results as closely as expected. Fortunately, this data provides an opportunity to increase the accuracy of the simulation for future control algorithm design and testing 


\section{CONCLUSION AND FUTURE WORK}

The two parameter adaptive control algorithm presented here yielded considerable reduction in steady-state displacement in both simulation and experiment for an externally applied sinusoidal load. Future enhancements to the current design will include four parameters to adjust in the output; the amplitude of the sine wave, the frequency of the sine wave, the phase shift, and the offset from zero. These enhancements will allow the algorithm to handle a constant disturbance, a sine wave oscillating about zero, or a sine wave of unknown or slowly varying frequency.

Ultimately, this type of algorithm will be applied to a magnetic bearing grinder. A picture of a grinder rotor/bearing system is shown in Figure 9. This system is currently installed in a test stand so that system identification and control algorithm testing can begin with an actual grinding system. This algorithm could help reduce unwanted motion of the spindle which leads to poor part finish and chatter.

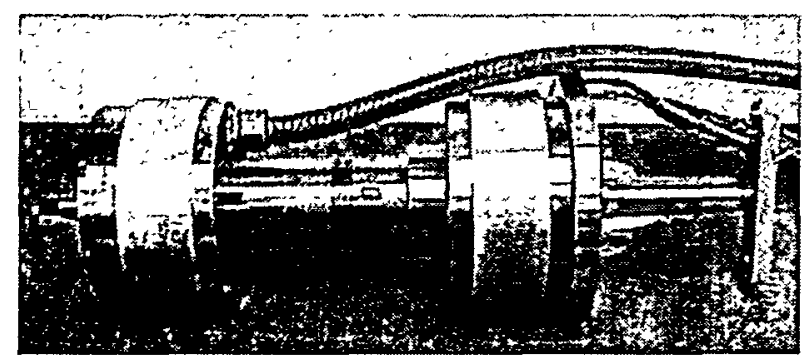

Figure 9. Magnetic rotor/bearing system for grinder

\section{ACKNOWLEDGEMENTS}

Sandia National Laboratories is a multiprogram laboratory operated by Sandia Corporation, a Lockheed Martin Company, for the United States Department of Energy under Contract DEAC04-94AL85000.

\section{REFERENCES}

Paden, B., "Operating Manual for the MBC500," Magnetic Moments, Inc., Santa Barbara CA.

Isermann, R. et al., Adaptive Control Systems, Prentice Hall, 1992.

Barney, P. et al., "Adaptive spindle balancing using magnetically levitated bearings", Proceedings of the 1999 IMECE. 